\title{
Determination of Heterogeneous Remote Sensing of the Earth System Rational Structure on Stage of External Design
}

\author{
Valeriy F. Volkov*a, Alexander S. Ponomareva, \\ Evgeniy N. Zhidkov ${ }^{\mathbf{b}}$ and Alexey A. Tolmachev \\ ${ }^{a}$ Military Space Academy \\ 13 Zhdanovskaya Str., Saint-Petersburg, 197198, Russia \\ ${ }^{b}$ Military Academy of Aero-Space Defense \\ named after the Marshal of Soviet Union G.K. Zhukov \\ 50 Zhigareva Str., Tver, 170022, Russia
}

Received 03.09.2018, received in revised form 28.09.2018, accepted 12.10.2018

It's suggested method of heterogeneous remote sensing of the Earth system rational structure determination. The method allow to define relation of space, manned and unmanned air reconnaissance systems in direct production in the interests of prescribed operability and quality of reconnaissance achievement with restrictions on resource intensity.

Keywords: remote sensing of the Earth system, efficiency, operability, life cycle, preference matrix, unmanned aircraft.

Citation: Volkov F.V., Ponomarev A.S., Zhidkov E.N., Tolmachev A.A. Determination of heterogeneous remote sensing of the Earth system rational structure on stage of external design, J. Sib. Fed. Univ. Eng. technol., 2018, 11(7), 789-800. DOI: $10.17516 / 1999-494 X-0094$.

(C) Siberian Federal University. All rights reserved

This work is licensed under a Creative Commons Attribution-NonCommercial 4.0 International License (CC BY-NC 4.0).

* Corresponding author E-mail address: volkov.v.f@mail.com, Ponomarev.a.s@mail.ru 


\title{
Определение рациональной структуры \\ разнородной системы дистанционного зондирования Земли
}

на этапе внешнего проектирования

\author{
В.Ф. Волков ${ }^{\mathrm{a}}$, А.С. Пономарев ${ }^{\mathrm{a}}$, \\ Е.Н. Жидков ${ }^{\tilde{0}}$, А.А. Толмачев ${ }^{\tilde{0}}$ \\ ${ }^{a}$ Военно-космическая академия имени А.Ф.Можайского \\ Россия, 197198, Санкт-Петербург, ул. Ждановская, 13 \\ ${ }^{6}$ Военная академия воздушно-космической обороньл \\ им. Маршала Советского Союза Г.К. Жукова \\ Россия, 170022, Тверь, ул. Жигарева, 50
}

В работе предложена методика обоснования рациональной структуры разнородной системы дистаниионного зондирования Земли (ДЗ3), позволяющая в прямой постановке для конкретных условий определять соотношение космических, пилотируемых и беспилотных средств ДЗЗ для обеспечения требуемой оперативности и качества мероприятий ДЗ3 при ограничениях на ресурсоемкость.

Ключевые слова: дистанционное зондирование Земли, эффективность, оперативность, жизненный цикл, матрица предпочтений, беспилотный летательный аппарат.

\section{Введение}

Анализ существующих систем дистанционного зондирования Земли (Д33) показал, что подобные системы не в полной мере отвечают предъявляемым к ним требованиям [12, 13]. Так, системы космического базирования способны обеспечить требуемую высокую оперативность сбора информации только при количестве космических аппаратов (КА) в группировке порядка нескольких сотен, а результативность функционирования пилотируемых и беспилотных авиационных средств ДЗ3 существенно зависит от метеоусловий и других факторов [5-7]. Для выхода из создавшейся проблемной ситуации в данной статье предлагается подход, состоящий в совместном и взаимоувязанном по времени и пространству применении авиационных и космических средств Д33, интегрированных в единую систему. Для проектирования такой разнородной системы ДЗ3 необходимо решить задачу обоснования оптимального соотношения ее структурных элементов. Подобные задачи относятся к классу многокритериальных оптимизационных задач с ограничениями, методы их решения (метод весовых коэффициентов, метод последовательных уступок, оптимизация по Парето, метод главного компонента, метод анализа иерархий) изложены в работах $[1-4,8,9]$.

На ранних стадиях жизненного цикла системы ДЗ3 наиболее целесообразно использовать метод анализа иерархий (МАИ). Данный метод состоит в декомпозиции решаемой задачи на более простые составные части и в дальнейшей обработке последовательности суждений лица, принимающего решение по парным сравнениям. Метод анализа иерархий включает в себя процедуры синтеза множественных суждений, получения приоритетности критериев и нахождения альтернативных решений. Такой подход к решению задачи выбора позволяет формализо- 
вать процесс логических рассуждений проектировщика системы, учесть внешние факторы и установить отношения между ними.

\section{Применение метода анализа иерархий для решения задачи} обоснования рациональной структуры разнородной системы ДЗ3

На первом шаге применения МАИ составляются матрицы приоритетов и попарных сравнений. Данные, включенные во все матрицы попарных сравнений, обрабатываются по формулам линейной алгебры в соответствии с алгоритмом, представленным в табл. 1 (исходная матрица помещена в левую часть таблицы).

Приоритеты синтезируются начиная со второго уровня вниз. Порядок синтеза состоит в следующем. Локальные приоритеты перемножаются на приоритеты соответствующего фактора на вышестоящем уровне и суммируются по каждому элементу в соответствии с факторами, на которые воздействует этот элемент.

Глобальные приоритеты позволяют путем сравнения принять окончательное решение. Для выполнения условий согласованности в матрицах попарных сравнений используются об-

ратные величины $a_{i j}=\frac{1}{a_{i j}}$ вместо традиционно используемых при построении интервальных шкал величин $a_{i j}=-a_{i j}$. Для контроля расчетов необходимо определять индекс согласованности (ИС), который может быть получен следующим образом. Сначала суммируется каждый столбец суждений, затем сумма первого столбца умножается на величину первой компоненты нормализованного вектора приоритетов, сумма второго столбца умножается на величину первой компоненты нормализованного вектора приоритетов, сумма второго столбца на вторую компоненту и т.д. Затем полученные числа суммируются. Таким способом можно получить

Таблица 1. Алгоритм проведения расчетов

Table 1. The algorithm of calculations

\begin{tabular}{|c|c|c|c|c|c|}
\hline$A_{1}$ & $\frac{\omega_{1}}{\omega_{1}} \frac{\omega_{1}}{\omega_{2}} \ldots \frac{\omega_{1}}{\omega_{12}} \frac{\omega_{1}}{\omega_{13}}$ & $\rightarrow$ & $\sqrt[13]{\frac{\omega_{1}}{\omega_{1}}} \frac{\omega_{1}}{\omega_{2}} \ldots \frac{\omega_{1}}{\omega_{12}} \frac{\omega_{1}}{\omega_{13}}$ & $\begin{array}{c}=a \\
+\end{array}$ & $\frac{a}{\text { сумма }}=x_{1}$ \\
\hline$A_{2}$ & $\frac{\omega_{2}}{\omega_{1}} \frac{\omega_{2}}{\omega_{2}} \ldots \frac{\omega_{2}}{\omega_{12}} \frac{\omega_{2}}{\omega_{13}}$ & $\rightarrow$ & $\sqrt[13]{\frac{\omega_{2}}{\omega_{1}} \frac{\omega_{2}}{\omega_{2}} \ldots \frac{\omega_{2}}{\omega_{12}} \frac{\omega_{2}}{\omega_{13}}}$ & $\begin{array}{c}=b \\
+\end{array}$ & $\frac{b}{\text { сумма }}=x_{2}$ \\
\hline$\ldots$ & $\ldots$ & $\ldots$ & $\ldots$ & $\ldots$ & $\ldots$ \\
\hline$A_{12}$ & $\frac{\omega_{12}}{\omega_{1}} \frac{\omega_{12}}{\omega_{2}} \ldots \frac{\omega_{12}}{\omega_{12}} \frac{\omega_{12}}{\omega_{13}}$ & $\rightarrow$ & $\sqrt[13]{\frac{\omega_{12}}{\omega_{1}} \frac{\omega_{12}}{\omega_{2}} \ldots \frac{\omega_{12}}{\omega_{12}} \frac{\omega_{12}}{\omega_{13}}}$ & $\begin{array}{c}=p \\
+\end{array}$ & $\frac{p}{\text { сумма }}=x_{12}$ \\
\hline$A_{13}$ & $\frac{\omega_{13}}{\omega_{1}} \frac{\omega_{13}}{\omega_{2}} \ldots \frac{\omega_{13}}{\omega_{12}} \frac{\omega_{13}}{\omega_{13}}$ & $\rightarrow$ & $\sqrt[13]{\frac{\omega_{13}}{\omega_{1}} \frac{\omega_{13}}{\omega_{2}} \ldots \frac{\omega_{13}}{\omega_{12}} \frac{\omega_{13}}{\omega_{13}}}$ & $\begin{array}{c}=q \\
+\end{array}$ & $\frac{q}{\text { сумма }}=x_{13}$ \\
\hline & & & & сумма & \\
\hline
\end{tabular}




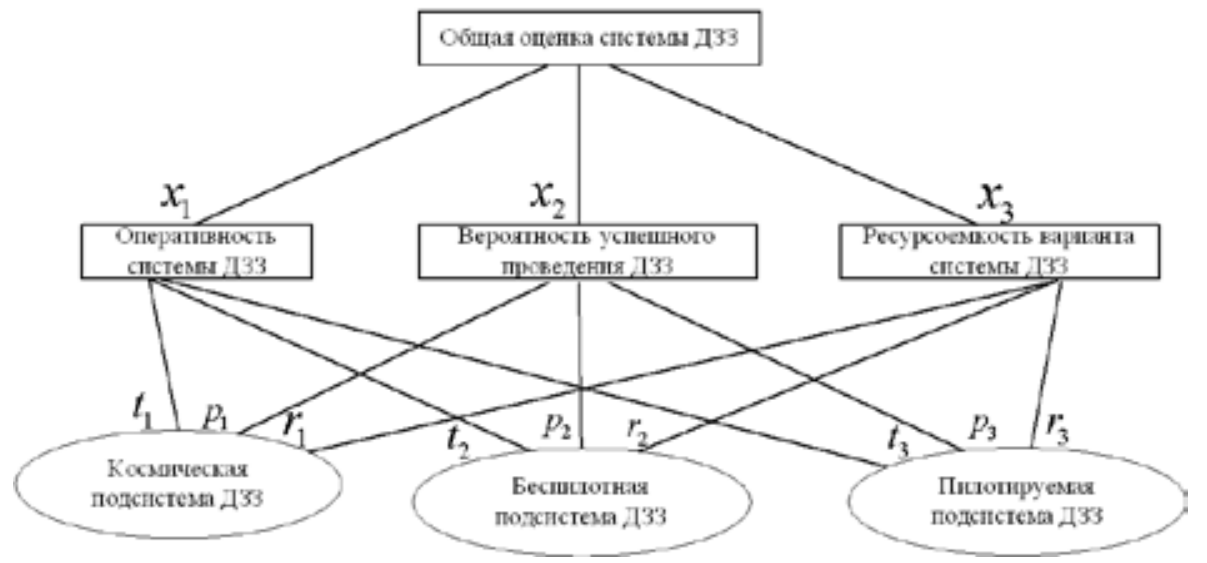

Рис. 1. Иерархия приоритетов

Fig. 1. Hierarchy of priorities

величину $\lambda_{\max }$. Для индекса согласованности имеем: ИС $=\left(\lambda_{\max }-n\right) /(n-1)$. Для обратносимметричной матрицы всегда $\lambda_{\max } \geq n$.

При реализации данного метода анализировались три типа зондирующих средств, входящих в качестве подсистем в проектируемую систему ДЗ3: космическая (КС), авиационная пилотируемая (АВ) и авиационная беспилотная (БПЛА). Для составления «матрицы предпочтений» использовались данные экспертного опроса специалистов в области ДЗ3. В опросе принимали участие 36 экспертов. Для оценивания достоверности полученных экспертных оценок был рассчитан коэффициент конкордации, его величина составляет 0,75 .

Далее были сформированы матрицы попарных сравнений частных показателей эффективности системы Д33, а именно оперативности, результативности и ресурсоемкости для различных вариантов разнородной системы. На рис. 1 представлена иерархия приоритетов перечисленных показателей, которые обозначены через $x_{1}, x_{2}$ и $x_{3}$ соответственно. Частные показатели оперативности, результативности и ресурсоемкости для каждой из подсистем обозначены буквами $t, p$ и $r$ соответственно.

Из вышеизложенного следует, что для получения общей оценки каждой подсистемы Дз3 необходимо умножить вес оценки этой шкалы по некоторому критерию на вес этого критерия и сложить значения, полученные для каждой системы по всем критериям. Общая оценка космической подсистемы вычисляется по формуле $k=t_{1}{ }^{*} x_{1}+p_{1}{ }^{*} x_{2}+r_{1}{ }^{*} x_{3}$, общая оценка авиационной подсистемы на базе БПЛА $-b=t_{2}{ }^{*} x_{1}+p_{2}{ }^{*} x_{2}+r_{2}{ }^{*} x_{3}$ и общая оценка авиационной подсистемы на базе пилотируемых ЛА $-a=t_{3}{ }^{*} x_{1}+p_{3}{ }^{*} x_{2}+r_{3}{ }^{*} x_{3}$.

Значение интегральной оценки всей разнородной системы ДЗЗ получается путем перемножения соответствующих матриц и транспонированных векторов-строк. При проведении исследований использовался генератор вариантов (комбинаций состава разнородной системы), описанный в работах $[6,7]$. При этом было введено ограничение «сверху» на число вариантов, полученное из экономических соображений. Всего были рассмотрены 17 комбинаций, 4 из которых были отброшены перед принятием окончательного решения как не представляющие интереса («нулевые» варианты). 


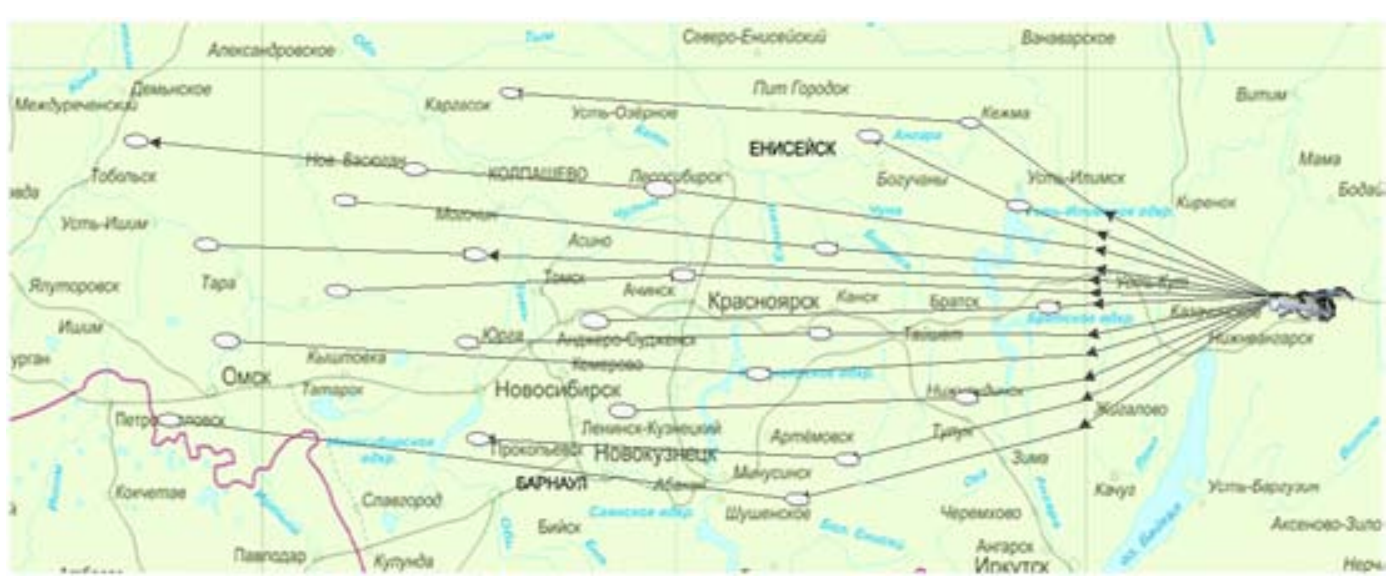

Рис. 2. Район ведения детального ДЗ3

Fig. 2. Area of detailed RSE

Таблица 2. Исходные данные о подсистемах ДЗ3

Table 2. Initial data on RSE subsystems

\begin{tabular}{|l|c|c|c|}
\hline & Оперативность, мин. & Результативность & Ресурсоемкость, у.е. \\
\hline $\begin{array}{l}\text { Космическая подсистема } \\
\text { ДЗ3 }\end{array}$ & $15-45$ & 0,95 & 973 \\
\hline $\begin{array}{l}\text { Пилотируемая подсистема } \\
\text { ДЗ3 }\end{array}$ & $110-120$ & 0,6 & 214 \\
\hline $\begin{array}{l}\text { Беспилотная подсистема } \\
\text { ДЗ3 }\end{array}$ & $70-80$ & 0,75 & 92 \\
\hline
\end{tabular}

В качестве района ДЗ3 рассматривался один из регионов с размерами 100 км $\times 300$ км (рис. 2).

Исходные данные для подсистем ДЗ3 по характеристикам оперативности функционирования, результативности (вероятности успешного проведения Д33) и ресурсоемкости (стоимости) приведены в табл. 2. Характеристики оперативности каждой из подсистем ДЗ3 заданы с учетом интервала времени на выход в район Д33 (рис. 2) (для ОГ КА) и на подготовку к проведению Д33 (для авиационных комплексов).

Матрица приоритетов различных показателей эффективности, сформированная экспертами, и результаты расчета вектора приоритетов представлены в табл. 3.

\section{Исходные данные для реализации МАИ и анализ результатов расчетов}

В табл. 4-6 представлены результаты расчетов векторов приоритетов по каждому показателю (оперативности, результативности, ресурсоемкости) для разных вариантов состава разнородной системы. Результаты расчетов интегрального показателя для каждого варианта отражены в табл. 7, на рис. 3 приведена соответствующая графическая зависимость интегрального показателя от номера варианта состава системы.

$$
-793-
$$


Таблица 3. Матрица приоритетов показателей оперативности, результативности, ресурсоемкости системы ДЗ3

Table 3. Matrix of priorities of indicators of efficiency, effectiveness, resource intensity of the RSE system

\begin{tabular}{|c|c|c|c|c|}
\hline & Оперативность & Результативность & Ресурсоемкость & Вектор \\
\hline Оперативность & 1 & 0,33 & 0,75 & 0,181378 \\
\hline Результативность & 3 & 1 & 3 & 0,598898 \\
\hline Ресурсоемкость & 1,33 & 0,33 & 1 & 0,219724 \\
\hline
\end{tabular}

Таблица 4. Матрица попарных сравнений по показателю оперативности для различных вариантов состава разнородной системы Д33

Table 4. Matrix of pairwise comparisons in terms of efficiency for different variants of the heterogeneous RSE system

\begin{tabular}{|c|c|c|c|c|c|c|c|}
\hline $\mathrm{k}: \mathrm{a}: \mathrm{b}$ & $2: 4: 2$ & $2: 3: 4$ & $2: 2: 6$ & $2: 1: 8$ & $1: 9: 2$ & $1: 8: 4$ & $1: 7: 6$ \\
\hline $2: 4: 2$ & 1,000 & 0,900 & 0,800 & 1,500 & 0,670 & 0,700 & 1,200 \\
\hline $2: 3: 4$ & 1,111 & 1,000 & 0,900 & 1,500 & 0,670 & 1,000 & 1,200 \\
\hline $2: 2: 6$ & 1,250 & 1,111 & 1,000 & 1,500 & 0,670 & 1,000 & 1,200 \\
\hline $2: 1: 8$ & 0,667 & 0,667 & 0,667 & 1,000 & 0,440 & 0,670 & 0,800 \\
\hline $1: 9: 2$ & 1,493 & 1,493 & 1,493 & 2,273 & 1,000 & 1,500 & 1,800 \\
\hline $1: 8: 4$ & 1,429 & 1,000 & 1,000 & 1,493 & 0,667 & 1,000 & 1,200 \\
\hline $1: 7: 6$ & 0,833 & 0,833 & 0,833 & 1,250 & 0,556 & 0,833 & 1,000 \\
\hline $1: 6: 8$ & 0,667 & 0,667 & 0,667 & 1,000 & 0,444 & 0,667 & 0,800 \\
\hline $1: 5: 10$ & 0,699 & 0,699 & 0,699 & 1,053 & 0,467 & 0,699 & 0,840 \\
\hline $1: 4: 12$ & 0,714 & 0,667 & 0,667 & 1,000 & 0,444 & 0,667 & 0,800 \\
\hline $1: 3: 14$ & 0,633 & 0,633 & 0,633 & 0,952 & 0,422 & 0,633 & 0,758 \\
\hline $1: 2: 16$ & 0,599 & 0,599 & 0,599 & 0,901 & 0,400 & 0,599 & 0,719 \\
\hline $1: 1: 18$ & 0,568 & 0,568 & 0,568 & 0,847 & 0,377 & 0,568 & 0,680 \\
\hline
\end{tabular}

Продолжение таблицы 4

Continuation of table 4

\begin{tabular}{|c|c|c|c|c|c|c|c|c|}
\hline $\mathrm{k}: \mathrm{a}: \mathrm{b}$ & $1: 6: 8$ & $1: 5: 10$ & $1: 4: 12$ & $1: 3: 14$ & $1: 2: 16$ & $1: 1: 18$ & & вектор \\
\hline $2: 4: 2$ & 1,500 & 1,430 & 1,400 & 1,580 & 1,670 & 1,760 & & 0,087 \\
\hline $2: 3: 4$ & 1,500 & 1,430 & 1,500 & 1,580 & 1,670 & 1,760 & & 0,092 \\
\hline $2: 2: 6$ & 1,500 & 1,430 & 1,500 & 1,580 & 1,670 & 1,760 & & 0,095 \\
\hline $2: 1: 8$ & 1,000 & 0,950 & 1,000 & 1,050 & 1,110 & 1,180 & & 0,061 \\
\hline $1: 9: 2$ & 2,250 & 2,140 & 2,250 & 2,370 & 2,500 & 2,650 & & 0,138 \\
\hline $1: 8: 4$ & 1,500 & 1,430 & 1,500 & 1,580 & 1,670 & 1,760 & & 0,095 \\
\hline $1: 7: 6$ & 1,250 & 1,190 & 1,250 & 1,320 & 1,390 & 1,470 & & 0,077 \\
\hline $1: 6: 8$ & 1,000 & 0,950 & 1,000 & 1,050 & 1,110 & 1,180 & & 0,062 \\
\hline $1: 5: 10$ & 1,053 & 1,000 & 1,050 & 1,105 & 1,170 & 1,235 & & 0,065 \\
\hline $1: 4: 12$ & 1,000 & 0,952 & 1,000 & 1,050 & 1,110 & 1,180 & & 0,062 \\
\hline $1: 3: 14$ & 0,952 & 0,905 & 0,952 & 1,000 & 1,055 & 1,118 & & 0,058 \\
\hline $1: 2: 16$ & 0,901 & 0,855 & 0,901 & 0,948 & 1,000 & 1,060 & & 0,055 \\
\hline $1: 1: 18$ & 0,847 & 0,810 & 0,847 & 0,894 & 0,943 & 1,000 & & 0,052 \\
\hline & & & & & & cyммa & 13,527 & \\
\hline
\end{tabular}


Таблица 5. Матрица попарных сравнений по показателю результативности для различных вариантов состава разнородной системы ДЗ3

Table 5. Matrix of pairwise comparisons in terms of performance for different variants of the heterogeneous RSE system

\begin{tabular}{|c|c|c|c|c|c|c|c|}
\hline $\mathrm{k}: \mathrm{a}: \mathrm{b}$ & $2: 4: 2$ & $2: 3: 4$ & $2: 2: 6$ & $2: 1: 8$ & $1: 9: 2$ & $1: 8: 4$ & $1: 7: 6$ \\
\hline $2: 4: 2$ & 1,000 & 1,000 & 1,000 & 1,330 & 1,670 & 1,670 & 1,330 \\
\hline $2: 3: 4$ & 1,000 & 1,000 & 1,000 & 1,330 & 1,670 & 1,670 & 1,330 \\
\hline $2: 2: 6$ & 1,000 & 1,000 & 1,000 & 1,330 & 1,670 & 1,670 & 1,330 \\
\hline $2: 1: 8$ & 0,752 & 0,752 & 0,752 & 1,000 & 1,250 & 1,250 & 1,000 \\
\hline $1: 9: 2$ & 0,599 & 0,599 & 0,599 & 0,800 & 1,000 & 1,000 & 0,800 \\
\hline $1: 8: 4$ & 0,599 & 0,599 & 0,599 & 0,800 & 1,000 & 1,000 & 0,800 \\
\hline $1: 7: 6$ & 0,752 & 0,752 & 0,752 & 1,000 & 1,250 & 1,250 & 1,000 \\
\hline $1: 6: 8$ & 0,752 & 0,752 & 0,752 & 1,000 & 1,250 & 1,250 & 1,000 \\
\hline $1: 5: 10$ & 0,752 & 0,752 & 0,752 & 1,000 & 1,250 & 1,250 & 1,000 \\
\hline $1: 4: 12$ & 0,752 & 0,752 & 0,752 & 1,000 & 1,250 & 1,250 & 1,000 \\
\hline $1: 3: 14$ & 0,752 & 0,752 & 0,752 & 1,000 & 1,250 & 1,250 & 1,000 \\
\hline $1: 2: 16$ & 0,752 & 0,752 & 0,752 & 1,000 & 1,250 & 1,250 & 1,000 \\
\hline $1: 1: 18$ & 0,752 & 0,752 & 0,752 & 1,000 & 1,250 & 1,250 & 1,000 \\
\hline
\end{tabular}

Продолжение таблицы 5

Continuation of table 5

\begin{tabular}{|c|c|c|c|c|c|c|c|c|}
\hline $\mathrm{k}: \mathrm{a}: \mathrm{b}$ & $1: 6: 8$ & $1: 5: 10$ & $1: 4: 12$ & $1: 3: 14$ & $1: 2: 16$ & $1: 1: 18$ & & вектор \\
\hline $2: 4: 2$ & 1,330 & 1,330 & 1,330 & 1,330 & 1,330 & 1,330 & & 1,331 \\
\hline $2: 3: 4$ & 1,330 & 1,330 & 1,330 & 1,330 & 1,330 & 1,330 & & 1,331 \\
\hline $2: 2: 6$ & 1,330 & 1,330 & 1,330 & 1,330 & 1,330 & 1,330 & & 1,331 \\
\hline $2: 1: 8$ & 1,000 & 1,000 & 1,000 & 1,000 & 1,000 & 1,000 & & 1,000 \\
\hline $1: 9: 2$ & 0,800 & 0,800 & 0,800 & 0,800 & 0,800 & 0,800 & & 0,799 \\
\hline $1: 8: 4$ & 0,800 & 0,800 & 0,800 & 0,800 & 0,800 & 0,800 & & 0,799 \\
\hline $1: 7: 6$ & 1,000 & 1,000 & 1,000 & 1,000 & 1,000 & 1,000 & & 1,000 \\
\hline $1: 6: 8$ & 1,000 & 1,000 & 1,000 & 1,000 & 1,000 & 1,000 & & 1,000 \\
\hline $1: 5: 10$ & 1,000 & 1,000 & 1,000 & 1,000 & 1,000 & 1,000 & & 1,000 \\
\hline $1: 4: 12$ & 1,000 & 1,000 & 1,000 & 1,010 & 1,000 & 1,000 & & 1,001 \\
\hline $1: 3: 14$ & 1,000 & 1,000 & 1,000 & 1,000 & 1,000 & 1,000 & & 1,000 \\
\hline $1: 2: 16$ & 1,000 & 1,000 & 1,000 & 1,000 & 1,000 & 1,000 & & 1,000 \\
\hline $1: 1: 18$ & 1,000 & 1,000 & 1,000 & 1,000 & 1,000 & 1,000 & & 1,000 \\
\hline & & & & & & сумма & 13,171 & \\
\hline
\end{tabular}


Таблица 6. Матрица попарных сравнений по показателю ресурсоемкости для различных вариантов состава разнородной системы ДЗ3

Table 6. Matrix of pairwise comparisons in terms of resource intensity for different variants of the heterogeneous RSE system

\begin{tabular}{|c|c|c|c|c|c|c|c|}
\hline $\mathrm{k}: \mathrm{a}: \mathrm{b}$ & $2: 4: 2$ & $2: 3: 4$ & $2: 2: 6$ & $2: 1: 8$ & $1: 9: 2$ & $1: 8: 4$ & $1: 7: 6$ \\
\hline $2: 4: 2$ & 1,000 & 1,010 & 1,020 & 1,030 & 0,970 & 0,920 & 0,990 \\
\hline $2: 3: 4$ & 0,990 & 1,000 & 1,010 & 1,021 & 0,960 & 0,913 & 1,010 \\
\hline $2: 2: 6$ & 0,980 & 0,990 & 1,000 & 1,010 & 0,950 & 0,904 & 0,968 \\
\hline $2: 1: 8$ & 0,971 & 0,979 & 0,990 & 1,000 & 0,940 & 0,895 & 0,985 \\
\hline $1: 9: 2$ & 1,031 & 1,042 & 1,053 & 1,064 & 1,000 & 0,952 & 1,020 \\
\hline $1: 8: 4$ & 1,087 & 1,095 & 1,106 & 1,117 & 1,050 & 1,000 & 1,071 \\
\hline $1: 7: 6$ & 1,010 & 0,990 & 1,033 & 1,015 & 0,980 & 0,934 & 1,000 \\
\hline $1: 6: 8$ & 1,003 & 0,980 & 1,022 & 1,033 & 0,971 & 0,924 & 0,990 \\
\hline $1: 5: 10$ & 0,990 & 1,002 & 1,012 & 1,024 & 0,962 & 0,916 & 0,980 \\
\hline $1: 4: 12$ & 0,980 & 0,993 & 1,012 & 1,013 & 0,952 & 0,906 & 0,971 \\
\hline $1: 3: 14$ & 0,971 & 0,982 & 0,992 & 1,002 & 0,942 & 0,897 & 0,961 \\
\hline $1: 2: 16$ & 0,962 & 0,972 & 0,982 & 0,992 & 0,932 & 0,887 & 0,951 \\
\hline $1: 1: 18$ & 0,952 & 0,962 & 0,971 & 0,980 & 0,923 & 0,878 & 0,941 \\
\hline
\end{tabular}

Продолжение таблицы 6

Continuation of table 6

\begin{tabular}{|c|c|c|c|c|c|c|c|c|}
\hline $\mathrm{k}: \mathrm{a}: \mathrm{b}$ & $1: 6: 8$ & $1: 5: 10$ & $1: 4: 12$ & $1: 3: 14$ & $1: 2: 16$ & $1: 1: 18$ & & вектор \\
\hline $2: 4: 2$ & 0,997 & 1,010 & 1,020 & 1,030 & 1,040 & 1,050 & 0,077 \\
\hline $2: 3: 4$ & 1,020 & 0,998 & 1,007 & 1,018 & 1,029 & 1,040 & & 0,077 \\
\hline $2: 2: 6$ & 0,978 & 0,988 & 0,988 & 1,008 & 1,018 & 1,030 & & 0,076 \\
\hline $2: 1: 8$ & 0,968 & 0,977 & 0,987 & 0,998 & 1,008 & 1,020 & & 0,075 \\
\hline $1: 9: 2$ & 1,030 & 1,040 & 1,050 & 1,062 & 1,073 & 1,084 & & 0,080 \\
\hline $1: 8: 4$ & 1,082 & 1,092 & 1,104 & 1,115 & 1,127 & 1,139 & & 0,084 \\
\hline $1: 7: 6$ & 1,010 & 1,020 & 1,030 & 1,041 & 1,052 & 1,063 & & 0,078 \\
\hline $1: 6: 8$ & 1,000 & 1,010 & 1,020 & 1,031 & 1,042 & 1,053 & & 0,077 \\
\hline $1: 5: 10$ & 0,990 & 1,000 & 1,010 & 1,020 & 1,031 & 1,053 & & 0,077 \\
\hline $1: 4: 12$ & 0,980 & 0,990 & 1,000 & 1,010 & 1,020 & 1,032 & & 0,076 \\
\hline $1: 3: 14$ & 0,970 & 0,980 & 0,990 & 1,000 & 1,010 & 1,021 & & 0,075 \\
\hline $1: 2: 16$ & 0,960 & 0,970 & 0,980 & 0,990 & 1,000 & 1,010 & & 0,074 \\
\hline $1: 1: 18$ & 0,950 & 0,950 & 0,969 & 0,979 & 0,990 & 1,000 & & 0,074 \\
\hline
\end{tabular}


Вывод: результаты расчетов показывают, что для исходных данных, представленных в табл. 2-6, рациональным вариантом разнородной системы ДЗ3 (табл. 7) является вариант № $3-N^{\mathrm{KC}}=2, N^{\mathrm{AB}}=2, N^{\text {БПлА }}=6$.

Таблица 7. Интегральная оценка вариантов разнородной системы ДЗ3

Table 7. Integrated assessment of options for heterogeneous RSE system

\begin{tabular}{|c|c|c|c|c|c|}
\hline \multirow{2}{*}{$№$ п/п } & \multicolumn{3}{|c|}{ Векторы приоритетов } & $\begin{array}{c}\text { Интегральная оценка варианта } \\
\text { системы Д33 } M_{\Sigma}\end{array}$ \\
\hline & & 0,181378 & 0,598898 & 0,219724 & \\
\hline 1. & $2: 4: 2$ & 0,087 & 1,331 & 0,077 & 0,8299 \\
\hline 2. & $2: 3: 4$ & 0,092 & 1,331 & 0,077 & 0,8307 \\
\hline 3. & $2: 2: 6$ & 0,095 & 1,331 & 0,076 & 0,8309 \\
\hline 4. & $2: 1: 8$ & 0,061 & 1,000 & 0,075 & 0,6266 \\
\hline 5. & $1: 9: 2$ & 0,138 & 0,799 & 0,080 & 0,5213 \\
\hline 6. & $1: 8: 4$ & 0,095 & 0,799 & 0,084 & 0,5142 \\
\hline 7. & $1: 7: 6$ & 0,077 & 1,000 & 0,078 & 0,6300 \\
\hline 8. & $1: 6: 8$ & 0,062 & 1,000 & 0,077 & 0,6270 \\
\hline 9. & $1: 5: 10$ & 0,065 & 1,000 & 0,077 & 0,6275 \\
\hline 10. & $1: 4: 12$ & 0,062 & 1,001 & 0,076 & 0,6273 \\
\hline 11. & $1: 3: 14$ & 0,058 & 1,000 & 0,075 & 0,6260 \\
\hline 12. & $1: 2: 16$ & 0,055 & 1,000 & 0,074 & 0,6253 \\
\hline 13. & $1: 1: 18$ & 0,052 & 1,000 & 0,074 & 0,6245 \\
\hline & & & & & $M_{\Sigma}^{\max }=0,8309$ \\
\hline
\end{tabular}

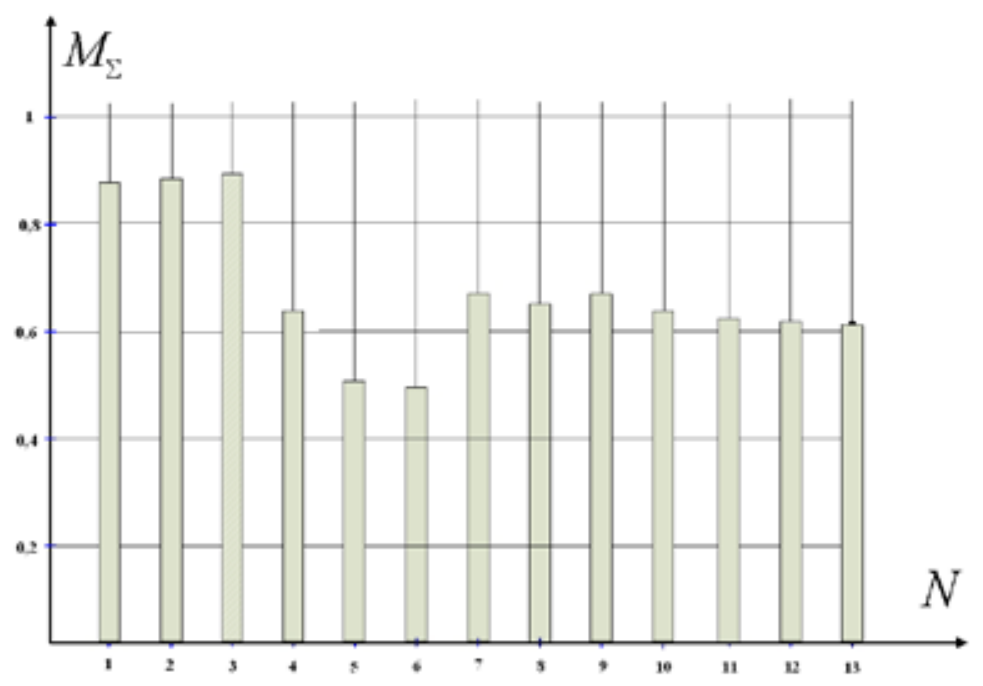

Рис. 3. Интегральные показатели эффективности системы Д3з

Fig. 3. Integrated performance indicators of the RSE system 
рЕзультаты

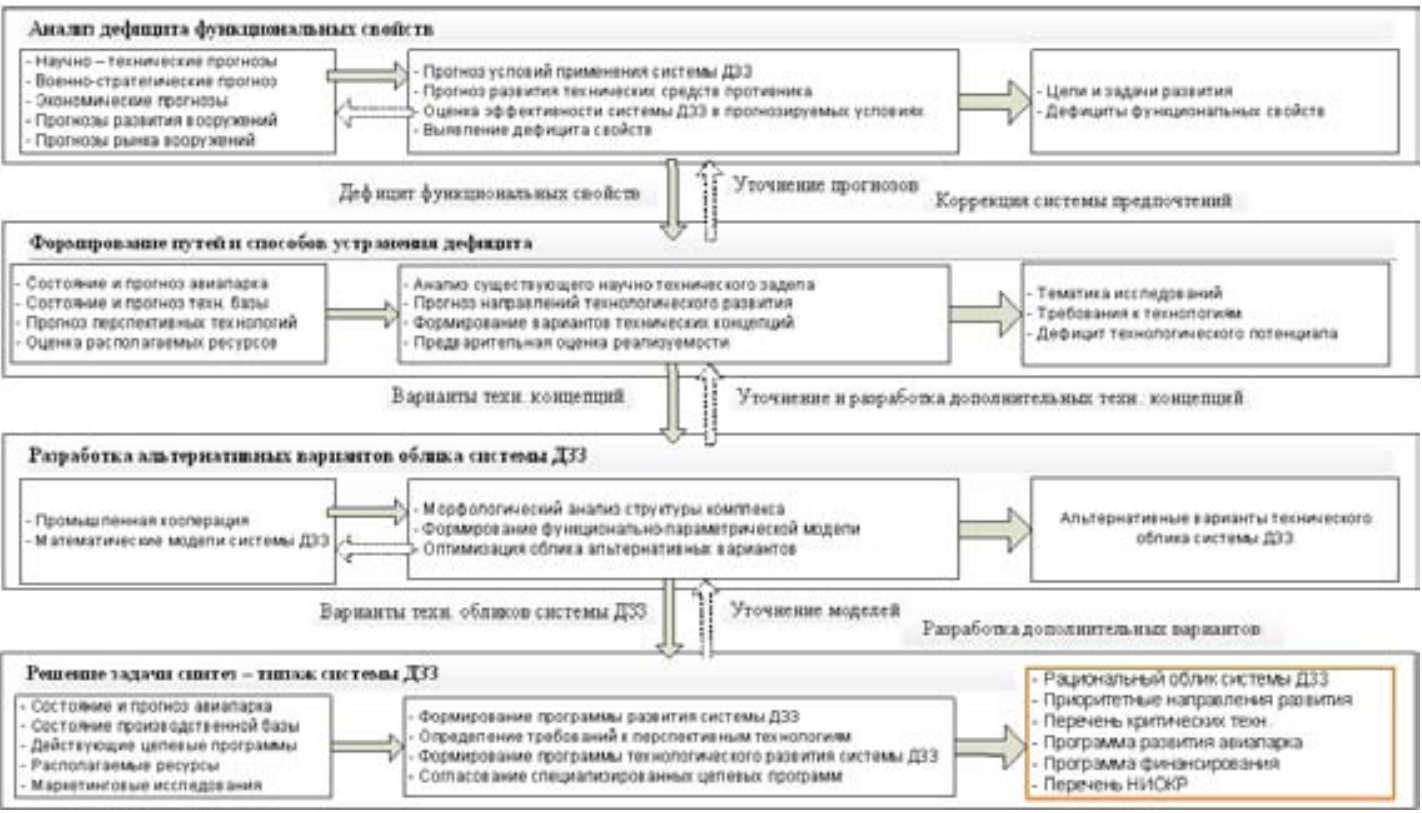

Рис. 4. Концептуальная схема технологии внешнего проектирования

Fig. 4. Conceptual scheme of external design technology

\section{Заключение}

Как уже отмечалось, метод анализа иерархий целесообразно использовать на ранних стадиях жизненного цикла, т.е. при решении задач внешнего проектирования. Концептуальная схема технологии внешнего проектирования изображена на рис. 4.

На конечных стадиях жизненного цикла целесообразно либо использовать игровые постановки [3, 4], либо применять метод сужения допустимых альтернатив [6]. Таким образом, метод анализа иерархий позволяет провести анализ проблемы. При этом проблема принятия решения представляется в виде иерархически упорядоченных:

а) главной цели (главного критерия) ранжирования возможных решений;

б) нескольких групп (уровней) однотипных факторов, так или иначе влияющих на рейтинг;

в) группы возможных решений;

г) системы связей, указывающих на взаимное влияние факторов и решений.

Метод позволяет провести сбор по проблеме; оценить противоречивость данных и минимизировать ее; провести синтез проблемы принятия решения; организовать обсуждение проблемы, способствует достижению консенсуса. Кроме того, следует отметить, что МАИ дает возможность оценить важность учета каждого решения и важность учета каждого фактора, влияющего на приоритеты решений, а также оценить устойчивость принимаемого решения.

В соответствии с формулировкой задачи принятия решения величина приоритета напрямую связана с оптимальностью решения. Поэтому решения с низкими приоритетами 
отвергаются как несущественные. Как отмечено выше, метод позволяет оценивать приоритеты факторов. Поэтому если при исключении некоторого фактора приоритеты решений изменяются незначительно, такой фактор можно считать несущественным для рассматриваемой задачи.

\section{Список литературы}

[1] Резников Б.А. Системный анализ иметоды системотехники. Часть 1. Методология системных исследований. Моделирование сложных систем. МО СССР, 1990. 522 c. [Reznikov B.A. System analysis and methods of system engineering. Part 1. The methodology of system research. Modeling of complex systems. MO SSSR, 1990. 522 p. (in Russia)]

[2] Вентцель Е.С. Исследование операщий. М.: Сов. радио, 1972. 565 с. [Ventcel' E.S. Research of operations. M.: Sov. radio, 1972. 565 p. (in Russia)]

[3] Динер И.Я. Исследование операщий: учебник, ч. ІІ. Л.: ВМОЛА им. А.Н. Крылова, 1968. 200 c. [Diner I.Ya. Research of operations: textbook, ch. II. L.: VMOLA im. A.N. Krylova, 1968. 200 p. (in Russia)]

[4] Солнышков Ю.С. Оптимизаџия выбора вооружения. М.: Воениздат, 1968. 104 с. [Solnyshkov Yu.S. Optimization of the choice of weapons. M.: Voenizdat, 1968. 104 p. (in Russia)]

[5] Фатеев В.Ф., Мальцев Г.Н., Минаков Е.П. и др. Малые космические аппараты информационного обеспечения. М.: Радиотехника, 2010. 316 c. [Fateev V.F., Mal'cev G.N., Minakov E.P. Small space vehicles for information support. M.: Radiotekhnika, 2010. 316 p. (in Russia)]

[6] Гнусарев Н.В. Геодезическое и баллистическое обеспечение космических систем дистанционного зондирования Земли. СПб.: ВКА имени А.Ф. Можайского, 2008. 220 с. [Gnusarev N.V. Geodetic and ballistic support of space systems for remote sensing of the Earth. $\mathrm{SPb}$.: VKA name after A.F. Mozhajskogo, 2008. 220 p. (in Russia)]

[7] Лаврова Н.П. Космическая фотосъемка. М., Недра, 1983. 300 с. [Lavrova N.P. Space photography. M., Nedra, 1983. 300 p. (in Russia)]

[8] Саати Т. Принятие решений. Метод анализа иерархий. М.: Радио и связь, 1993. 278 с. [Saati T. Decision making. Hierarchy analysis method. M.: Radio i svyaz', 1993. 278 p. (in Russia)]

[9] Москвин Б.В., Козлов Ю.В. Математическая модель оценивания потенциильных возможностей системы управления при обнаружении противника в районе боевых действий. Космическая кибернетика: Монография. СПб.: ВИКУ, 2003. 183-188. [Moskvin B.V., Kozlov Yu.V. Mathematical model of estimation of potential possibilities of control system at detection of the opponent in the area of military operations // Kosmicheskaya kibernetika: Monografiya. SPb.: VIKU, 2003. 183-188 (in Russia)]

[10] Ваулин А.Е., Волков В.Ф. Общая методология решения задач оптимизации проиесса обслуживания подвижных объектов как задач комбинаторного анализа. Депонированная рукопись № 228. М.: ЦИВТИ МО СССР, 1979. 24 с. [Vaulin A.E., Volkov V.F. General methodology for solving problems of optimization of the process of maintenance of mobile objects as problems of combinatorial analysis. Deponirovannaya rukopis' No. 228. M.: CIVTI MO SSSR, 1979. 24 p. (in Russia)]

[11] Волков В.Ф., Поляков С.А. Генерация вариантов обслуживания: сборник алгоритмов и программ типовых задач, выпуск 8. Л.: МО СССР, 1987. 71-79 [Volkov V.F., Polyakov S.A. 
Generating service options: sbornik algoritmov i programm tipovyh zadach, vypusk 8. L.: MO SSSR, 1987. 71-79 (in Russia)].

[12] Поповкин В.А. Показатели и критерии сбалансированности развития космического информационного обеспечения. Журнал Стратегическая стабильность. М.: Изд. Академии военных наук, 2004, 4, 21-27. [Popovkin V.A. Indicators and criteria for balanced development of space information support. J. Strategic stability. M.: Pub. Academy of military Sciences, 2004, 4, 21-27 (in Russia)]

[13] Беспилотные аппараты: способы применения в составе боевых систем. Журнал Военная мыссль, 2012 , 3, 39-42. [Unmanned vehicles: methods of use in combat. J. Military thought, 2012, 3, 39-42 (in Russia)] 